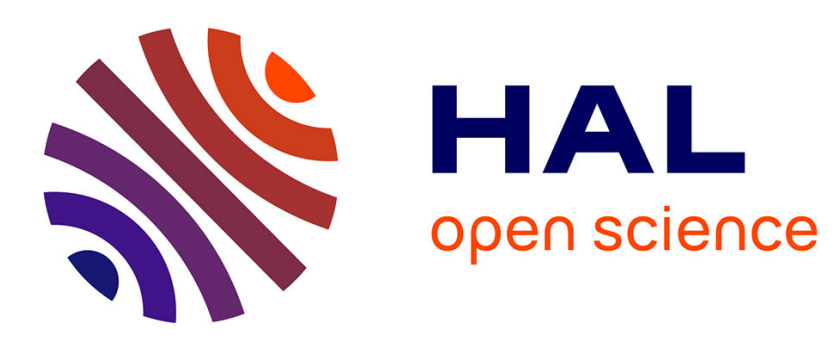

\title{
Nano-shaped hot-wire for ultra-high resolution anemometry in cryogenic helium
}

Pantxo Diribarne, Pierre Thibault, Philippe-Emmanuel Roche

\section{To cite this version:}

Pantxo Diribarne, Pierre Thibault, Philippe-Emmanuel Roche. Nano-shaped hot-wire for ultra-high resolution anemometry in cryogenic helium. Review of Scientific Instruments, 2019, 90 (10), pp.105004. $10.1063 / 1.5116852$. cea-02282964v3

\section{HAL Id: cea-02282964 https://hal-cea.archives-ouvertes.fr/cea-02282964v3}

Submitted on 15 Oct 2019

HAL is a multi-disciplinary open access archive for the deposit and dissemination of scientific research documents, whether they are published or not. The documents may come from teaching and research institutions in France or abroad, or from public or private research centers.
L'archive ouverte pluridisciplinaire HAL, est destinée au dépôt et à la diffusion de documents scientifiques de niveau recherche, publiés ou non, émanant des établissements d'enseignement et de recherche français ou étrangers, des laboratoires publics ou privés. 


\title{
Nano-shaped hot-wire for ultra-high resolution anemometry in cryogenic helium
}

\author{
Cite as: Rev. Sci. Instrum. 90, 105004 (2019); doi: 10.1063/1.5116852 \\ Submitted: 28 June 2019 • Accepted: 6 September 2019 • \\ Published Online: 14 October 2019
}

Pantxo Diribarne, ${ }^{1,2, a)}$ (D) Pierre Thibault, ${ }^{1,2}$ and Philippe-Emmanuel Roche ${ }^{1}$ (D)

AFFILIATIONS
${ }^{1}$ Institut NEEL, CNRS, Université Grenoble Alpes, F-38042 Grenoble, France
${ }^{2}$ dSBT/IRIG CEA, Université Grenoble Alpes, F-38054 Grenoble, France

${ }^{a)}$ Electronic mail: pantxo.diribarne@univ-grenoble-alpes.fr

\begin{abstract}
We present the principle, modeling, and the first implementation of a new type of high resolution hot-wire anemometer designed to operate at cryogenic temperatures and very high Reynolds numbers. Its spatial resolution of a few micrometers is comparable to the most spatially resolved hot-wires reported in the literature. Compared to existing designs, its fabrication involves a limited number of steps, essentially the shaping at nanoscales of a superconducting NbTi wire of submicron diameter. The velocity spectra in the far wake of a centimeter-sized grid are measured as a proof of concept in stringent flow conditions.
\end{abstract}

Published under license by AIP Publishing. https://doi.org/10.1063/1.5116852

\section{INTRODUCTION}

Among turbulent flows, the most intense ones are characterized by the widest separation between the typical sizes $L$ and $\eta$ of the largest and the smallest eddies. ${ }^{1}$ The dimensionless Reynolds number $(R e)$ is commonly used to quantify turbulence intensity, and it is directly related to this size ratio $R e \simeq(L / \eta)^{4 / 3}$. Very large Reynolds numbers (say $R e \gtrsim 10^{6}$ ) are met in various environmental and industrial flows and are of special interest to models accounting for the universal scaling properties of turbulence when $R e \rightarrow \infty$.

Such intense turbulence levels can also be produced in the laboratory using fluids of very low viscosity. Most often, cryogenic helium is chosen for its favorable properties, ${ }^{2}$ either in its liquid (see Refs. 3 and 4 ) or gaseous state (see Refs. 5-7), but large-scale room-temperature facilities can also reach very high Re using pressurized air (see Ref. 8) and pressurized $\mathrm{SF}_{6}$ (see Ref. 9). Unfortunately, the wide separation in eddy sizes in these laboratory flows, e.g., 4.5 decades for $R e \simeq 10^{6}$, implies that the smallest eddies are often tinier than $10 \mu \mathrm{m}$, that is, out-of-reach of the best commercial anemometers, by two-decades.

Thus, over the last decades, significant efforts have been dedicated to the development of high-resolution thermal anemometers for very high $R e$ turbulence experiments. Thermal anemometers with a spatial resolution from $25 \mu \mathrm{m}$ down to few microns have been hand-made specifically for cryogenic flows, either using carbon fibers ${ }^{5}$ or glass fibers coated with metals or a superconductor. ${ }^{7,10,11}$ Some fully microfabricated anemometers with a spatial resolution of order $50 \mu \mathrm{m}$ have been recently developed in Princeton ${ }^{12,13}$ and Tel Aviv ${ }^{14}$ for room temperature operation, and others with a spatial resolution of a few microns have recently been reported in Grenoble, both for room and cryogenic temperatures.

The present study proposes a new concept for thermal anemometry in cryogenic helium flows, typically below $5 \mathrm{~K}$. It relies on a submicron superconducting fiber shaped with a focused ion beam [see Fig. 1(b)]. Compared to the previous designs, it benefits from a significantly reduced number of fabrication steps while preserving the record resolution of few microns achieved by other probes.

\section{OPERATING PRINCIPLE}

Like in traditional hot-wire anemometers, the velocity measurement principle is based on the overheating of the central region of a wire of submicron diameter (here $D \simeq 400 \mathrm{~nm}$ ), held on a supporting fork facing the flow [see Fig. 1(a)]. Unlike traditional hotwires, the wire material is $\mathrm{NbTi}$, which has a superconducting state below $T_{c} \simeq 10 \mathrm{~K}$ and a normal (resistive) state above this transition temperature. 
a)

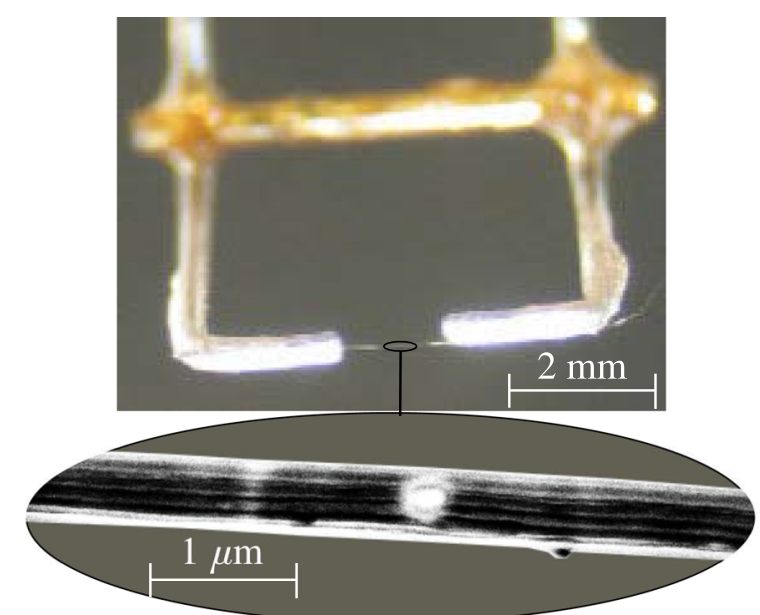

c)

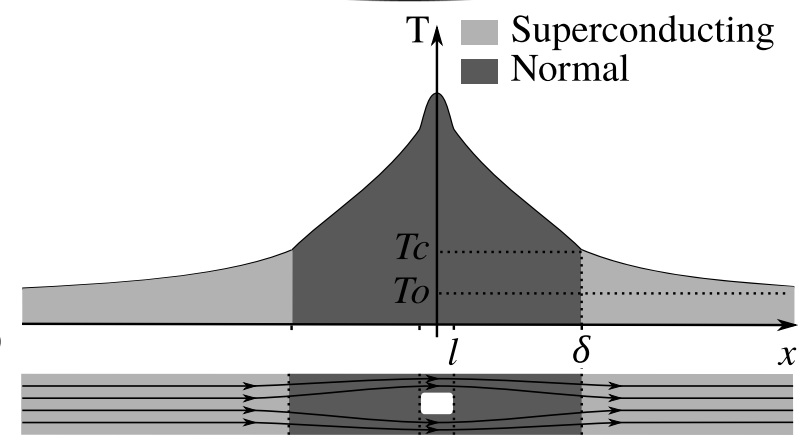

(1) (2)

FIG. 1. Illustration and images of the hot-wire. (a) Picture of the wire stretched between the tips of the prongs. (b) Scanning electron microscopy picture of the $\mathrm{NbTi}$ wire with its reduced cross section drilled using a focused ion beam (central rectangular hole). (c) Typical temperature profile along the wire and schematic of the three regions of the wire in nominal operating conditions: (1) normal state with reduced cross section, (2) normal state, and (3) superconducting state.

In nominal operation conditions, the central region of the wire is kept in its normal state $\left(>T_{c}\right)$ by the Joule effect, while the outer part of the wire remains in a superconducting state, thanks to the cooling by the flow of temperature $T_{0}<T_{c}$. Thus, the total electrical resistance $R$ of the wire is fully determined by the length $\delta$ of the central region of the wire.

The length $\delta$ of the normal region, and therefore the wire resistance $R$, depends on the balance between the Joule effect and the efficiency of the cooling process (forced convection), and it thus directly relates to the velocity of the flow.

This measuring principle is inspired from previous works by Castaing and co-workers. ${ }^{10}$ A key difference of the current design is that the central region, which ignites the superconducting to normal transition, is created mechanically by a local reduction in the cross section of a superconducting wire and not by means of a resistive coating surrounded by superconducting coatings on a glass fiber.

In the absence of an electrical current, the wire is entirely superconducting at its working temperature (here $T_{0} \lesssim 5 \mathrm{~K}$ ) and a current must ignite the transition from the superconducting state to

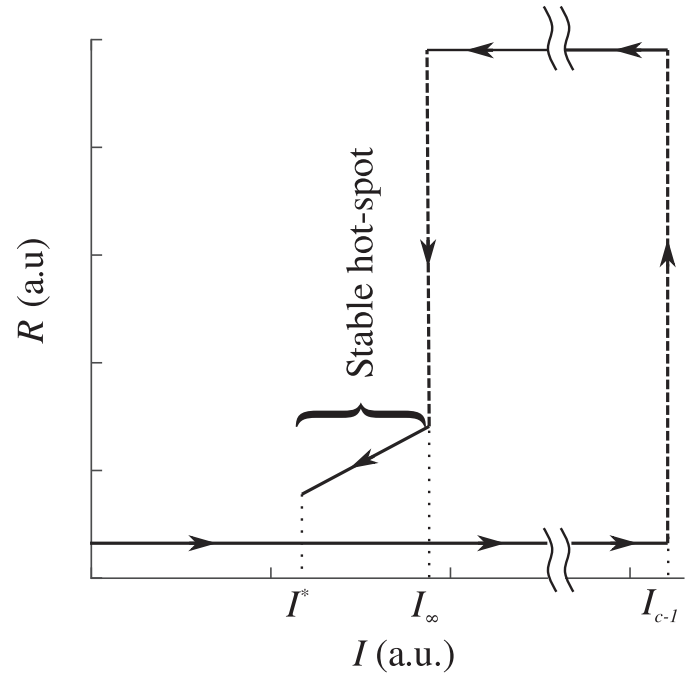

FIG. 2. Sketch of the current cycle that is needed in order to ignite the hot spot: the hot-wire is submitted to a fast pulse of current in order to pass the critical current and finally only leave the central region in the normal state.

the normal state in the central region (see Fig. 2). For this purpose, the wire is driven with a current greater than $I_{c-1}\left(T_{0}\right)$, the critical current of the central region at temperature $T_{0}$. For such a current, the first appearance of a resistive/dissipative section triggers a global transition of the whole wire to the normal state. In order to let most of the wire recover its superconducting state, and to get a stable hot-spot in the central region only, the current is then decreased below a current $I_{\infty} \ll I_{c-1}\left(T_{0}\right)$. In the superconducting magnet literature (see Dresner's review ${ }^{16}$ ), $I_{\infty}$ is the maximum current to achieve cryostability, i.e., the current below which the cable will recover its superconducting state, whatever the extent of the perturbation.

In practice, the ignition of the hot spot is obtained by first choosing a current $I \lesssim I_{\infty}$ and then by submitting the wire to a rapid pulse of current $\delta I \approx I_{c-1}$.

\section{MANUFACTURING}

The original NbTi wires are extracted from a multifilament cable. The main stages of manufacturing involve the isolation of a single wire, the electrical contacting between two prongs, and finally the local etching to reduce the wire cross section.

The multifilament cable we use is made of about one million NbTi nontwisted filaments with diameters in a range $0.4 \mu \mathrm{m}<D$ $<1 \mu \mathrm{m}$, enclosed in a copper matrix. The matrix is eliminated by dipping the bare cable into a $35 \%$ nitric acid solution. While the copper dissolves, the NbTi wires remain intact. The tangle of NbTi filaments is then rinsed with water and spread on a glass plate so as to ease the wire separation after drying. The next stage consists in manually extracting a single wire, using fine tweezers under a binocular magnifier. The wire is then stretched between the prongs of the definitive support [see Fig. 1(a)].

The next stage is the electrical contacting which requires a great care to prevent the resistive dissipation that may occur at the 


\section{Saw Cut}

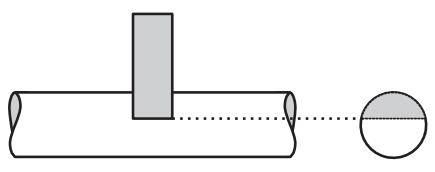

Directional Erosion

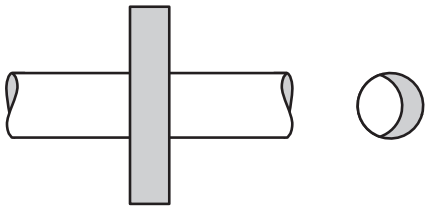

Hole Drilling

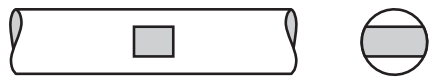

FIG. 3. Schematic of the three main etching shapes that were realized. Between the 3 shapes: (i) saw cut, (ii) directional erosion, and (iii) hole drilling, (iii) gave the best results.

contacts between the wire and the prongs and that would lead to additional and undesired hot spots. The wire surface is thus first cleaned with an Ar plasma. A thick layer (in general $1 \mu \mathrm{m}$ ) of aluminum or gold is then deposited by evaporation under secondary vacuum. During this operation, the central region of the wire is protected against metal deposition by a $1 \mathrm{~mm}$ wide mechanical mask so that the sensitive part of the wire is not short-circuited by the metal.

Eventually, the reduction in the cross section of the wire in the central region is achieved using a gallium-based Focused Ion Beam (FIB). We first take a picture of the wire and select graphically the rectangular area to be etched. We then proceed with the actual gallium beam etching which lasts only a few seconds. Three etching shapes have been tested (see the left side of Fig. 3): a "saw-cut" lateral etching, a directional erosion of the top surface, and a squareshaped-hole drilling through the wire. From the mechanical resistance point of view, the hole was found to be the most satisfactory shape. It allows for the removal of matter in the center of the wire, where the mechanical constraints are smaller than in the outer part.

The size of the hole must be chosen carefully. As expected, large holes lead to mechanically fragile sensors. On the other hand, large holes are more favorable from the thermal stability point of view, as will be shown in Sec. V. We found empirically that the largest holes that led to workable sensors had dimensions $D / 2 \times D / 2$, where $D$ is the diameter or the wire. This results in a section ratio $\alpha=\mathcal{S}_{\text {raw }} / \mathcal{S}_{\text {etched }} \approx 2.56$.

\section{CALIBRATION CURVE AT CONSTANT CURRENT}

The results discussed in this section were obtained with a wire $500 \mathrm{~nm}$ in diameter. The hole cross section is about $500 \mathrm{~nm}$ long by $250 \mathrm{~nm}$ large as estimated from the scanning electron microscope (SEM) pictures. From those values, the section ratio can be estimated to be $2<\alpha<2.5$.
We use liquid helium $\left(T_{0}=3.3 \mathrm{~K}\right)$ as the working fluid. Its pressure is kept higher than the critical pressure $P_{\text {crit }} \approx 2.2$ bars to avoid the onset of boiling at the surface of the wire.

The wire is tested in a small grid flow driven by a cryogenic pump allowing to reach velocities up to about $4.6 \mathrm{~m} / \mathrm{s}$ in a tube of diameter $5 \mathrm{~mm}$. The calibration is done at constant current using the procedure described in Sec. II with $\delta I=6 \mathrm{~mA}$ current pulses. For each velocity, we first find the minimal current allowing a hot spot to self-sustain after a current pulse; we then increase the current and measure the resulting voltage.

Figure 4 shows the resistance $R$ (right axis) as a function of the current $I$ (x-axis) for various velocities $v$. From resistance values, the length of the hot spot is inferred (see left axis) using the following relationship:

$$
R=2\left(l r_{1}+(\delta-l) r_{2}\right)
$$

where $r_{2} \approx 4.8 \Omega / \mu \mathrm{m}$ is the resistance per unit length of the raw wire in its normal state, $r_{1}=\alpha r_{2}$ is the estimated resistance per unit length of the reduced section area, and $l \approx 250 \mathrm{~nm}$ is the half-length of the hole and $\delta$ is the half-length of the hot-spot.

At null forced velocity, no stable hot-spot can be obtained: after a current pulse, the hot-wire returns to superconducting state for $I \lesssim 0.295 \mathrm{~mA}$ and remains entirely normal for $I \gtrsim 0.295 \mathrm{~mA}$.

After turning on the flow, stable hot-spots are observed from the smallest achievable velocity, $v=1.6 \mathrm{~m} / \mathrm{s}$, up to the largest one, $v=4.6 \mathrm{~m} / \mathrm{s}$. For a given velocity, we denote $I^{*}$ the smallest current at which a stable hot-spot is observed and $\delta^{*}$ the corresponding length of the hot-spot. The length of the hot-spot increases with the current

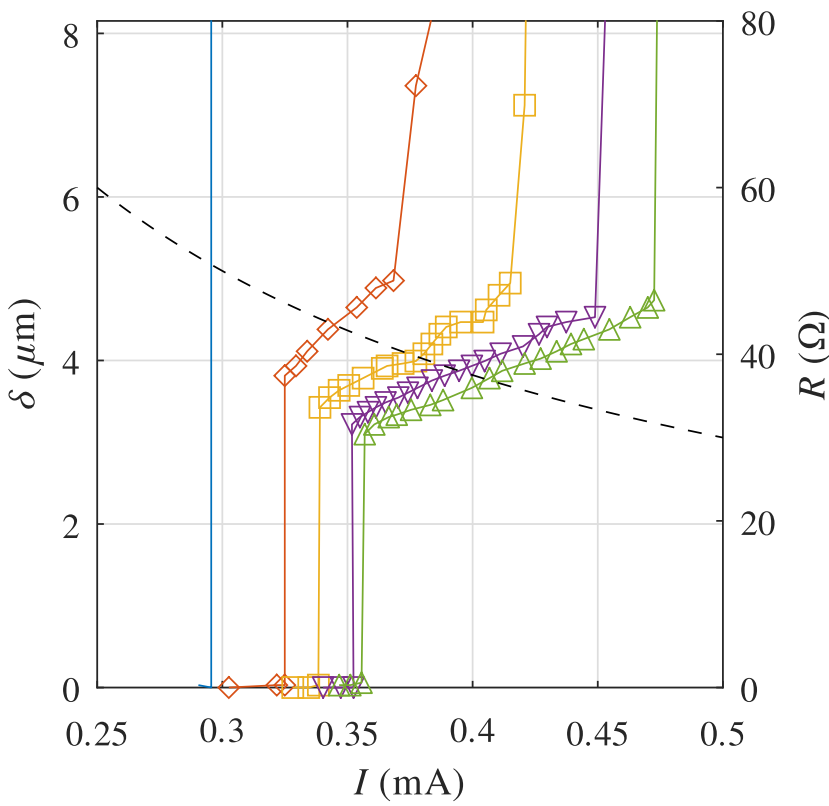

FIG. 4. Measured resistance of the wire (right axis) and corresponding estimated hot-spot length (left axis) as a function of the current for various velocities: $0 \mathrm{~m} / \mathrm{s}$ (no symbol), $1.6 \mathrm{~m} / \mathrm{s}$ (red diamond), $2.3 \mathrm{~m} / \mathrm{s}$ (yellow square), $3.4 \mathrm{~m} / \mathrm{s}$ (violet trian$\mathrm{gle}$ ), and $4.6 \mathrm{~m} / \mathrm{s}$ (green triangle). The dashed line indicates the hot-spot length (and resistance) for a fixed driving voltage $V=15 \mathrm{mV}$ across the wire. 
up to a current $I_{\infty}$ at which the wire transits entirely to its normal state.

As the velocity is increased, both $I^{*}$ and $I_{\infty}$ increase, but the latter faster than the former, so that the range of currents for which the hot-spot is stable tends to broaden with velocity.

The minimal half-length of the hot-spot $\delta^{*}$, which can be seen as the spatial resolution of the sensor, is generally one order of magnitude larger than $l$, the half-length of the reduced section area. Note also that $\delta^{*}$, and thus the best achievable resolution, decreases as the velocity is increased.

\section{THERMAL EXCHANGE MODEL}

To model the hot wire response, we make three main hypotheses. First, the temperature of the wire is assumed to be homogeneous across its section so that the temperature profile is only a function of the longitudinal dimension $x$ (1D hypothesis). Second, we assume that the wire response time is much shorter than velocity fluctuation time scales so that the wire is always in thermal equilibrium (quasi-static hypothesis). Both hypotheses are common in cryogenic thermal microanemometry and result from the vanishingly small heat capacity of most solids as compared to liquid helium at low temperature. ${ }^{7,10,11,17,18}$ Third, we assume that the sensor can be divided into three regions with the following properties [see Fig. 1(c)]:

- The central region (1) of length $2 l$ which has a reduced cross section. In nominal conditions, this region is in normal state and its resistance per unit length is $r_{1}$.

- The region (2) on both sides of the central region, which is also in normal state in nominal conditions and has a resistance per unit length $r_{2}$ and length $\delta-l$ each. Depending on the current, this region may or may not exist.

- The outer region (3). In nominal conditions, the wire is in its superconducting state and shows no resistance $\left(r_{3}=0\right)$.

Given a wire of raw diameter $D$, one can compute the resistances per unit length as follows: $r_{2}=4 \rho_{\mathrm{NbTi}} / \pi D^{2}=r_{1} / \alpha$, where $\rho_{\mathrm{NbTi}}$ is the electrical resistivity of $\mathrm{NbTi}$ in the normal state.

The local thermal equilibrium in each region can then be written as a balance between a Joule heat source, a conductive heat flux along the wire, and a convective heat flux to the fluid,

$$
r_{i} I^{2}=-K_{i} \frac{d^{2} T}{d x^{2}}+K_{h}\left(T-T_{0}\right)
$$

where $i \in[1,2,3]$ and $K_{i}=k_{\mathrm{NbTi}} \mathcal{S}_{i} . k_{\mathrm{NbTi}}$ is the thermal molecular conductivity of the wire, and $\mathcal{S}_{i}$ is the cross section of the considered region. $K_{h}=\pi k_{\mathrm{He}} \mathrm{Nu}$, where $\mathrm{Nu}$ is the Nusselt number that depends on the velocity of the fluid around the wire.

A similar equation was already discussed by Castaing in a previous hot-wire study, ${ }^{10}$ and its resolution can be formally ported to the present case. Thus, the resolution of this equation leads to a temperature profile of the form

$$
T(x)=T_{0}+\frac{r_{i} I^{2}}{K_{h}}+\Delta_{+} e^{x / \Lambda_{i}}+\Delta_{-} e^{-x / \Lambda_{i}},
$$

where $\Lambda_{i}=\sqrt{K_{i} / K_{h}}$ is the characteristic spreading length of the hot spot in region $i$ and where $\Delta_{+}$and $\Delta_{-}$are constants that depend on the boundary conditions.

At low current, the temperature at $x=l$ is below $T_{c}$, and only regions (1) and (3) exist. The current $I_{l}$ at which $T(l)=T_{c}$ is obtained from Eq. (3),

$$
I_{l}=\left[\frac{K_{h}\left(T_{c}-T_{0}\right)}{r_{1}}\left(1+\frac{\Lambda_{3}}{\Lambda_{1}} \operatorname{coth} \frac{l}{\Lambda_{1}}\right)\right]^{1 / 2} .
$$

In principle, the resolution of Eq. (3) should take into account the fact that the critical temperature itself is a function of the current. Such a correction is left for future improvements, and we have assumed that $T_{c} \approx T_{c}(I=0)$.

Note that, as will be shown later, this current $I_{l}$ is not necessarily the smallest current at which region (2) may exist or not correspond to a stable state.

In any event, the equilibrium current $I_{y}$ as a function of the normalized length of region (2), $y=(\delta-l) / \Lambda_{2}$, is determined by the condition $T(\delta-l)=T_{c}$ in Eq. (3). Some simple analytics lead to

$$
I_{y}=I_{l} e^{y / 2}\left[1+\frac{r_{2}}{r_{1}}\left(\frac{\Lambda_{3}}{\Lambda_{1}} \operatorname{coth} \frac{l}{\Lambda_{1}} \sinh y+2 \sinh ^{2} \frac{y}{2}\right)\right]^{-1 / 2} .
$$

Finally, beyond a threshold current $I_{\infty}$, no stable hot-spot can be observed as the length of region (2) extends to infinity,

$$
I_{\infty}=\lim _{y \rightarrow \infty} I_{y}=\left[\frac{2 K_{h}\left(T_{c}-T_{0}\right)}{r_{2}}\right]^{1 / 2} .
$$

Although the mathematical formulation of this model is similar to the previous model by Castaing et al., ${ }^{10}$ the value of the parameters greatly differs, in such a way that Eq. (5) now corresponds to both stable and unstable solutions, which were not studied in previous papers. The main difference with previous cryogenic hot-wires design lies in the resistance ratio $\alpha=r_{1} / r_{2}$ which is here necessarily small. Indeed, as previously mentioned, due to mechanical constraints, it is unsafe to reduce the wire section by a factor larger than $\alpha \approx 2.5$. When $\alpha$ is large enough, the current $I_{y}$ increases monotonically with the normalized length of region (2), $y$, which means $d I_{y} / d y>0$. The latter is not always true for arbitrary values of $\alpha$. The stability condition $d I_{y} / d y>0$ is only met for transited lengths larger than a threshold $\delta^{*}$. Using Eq. (5), one finds for the stability limit

$$
\delta^{*}=l+\Lambda_{2} \log \left[\frac{\alpha^{1 / 2} \operatorname{coth}\left(\alpha^{1 / 2} l / \Lambda_{2}\right)-1}{\alpha-1}\right] .
$$

Here, $2 \delta^{*}$ is the length of the smallest achievable hot spot with a given section ratio $\alpha$ and Nusselt number $N u$.

In Fig. 5, we have represented the length of the normal region $\delta$ as function of the current $I$ according to Eq. (5), for variable section ratios (top) and Nusselt numbers (bottom). Helium and $\mathrm{NbTi}$ properties were taken from the literature. ${ }^{19}$ The thermal conductivity of $\mathrm{NbTi}$ varies almost one decade over the temperature range of interest, ${ }^{20}$ and we chose the average value in this temperature range.

Even though this model is an approximation of the actual physics, it clearly succeeds in capturing most of the features observed 

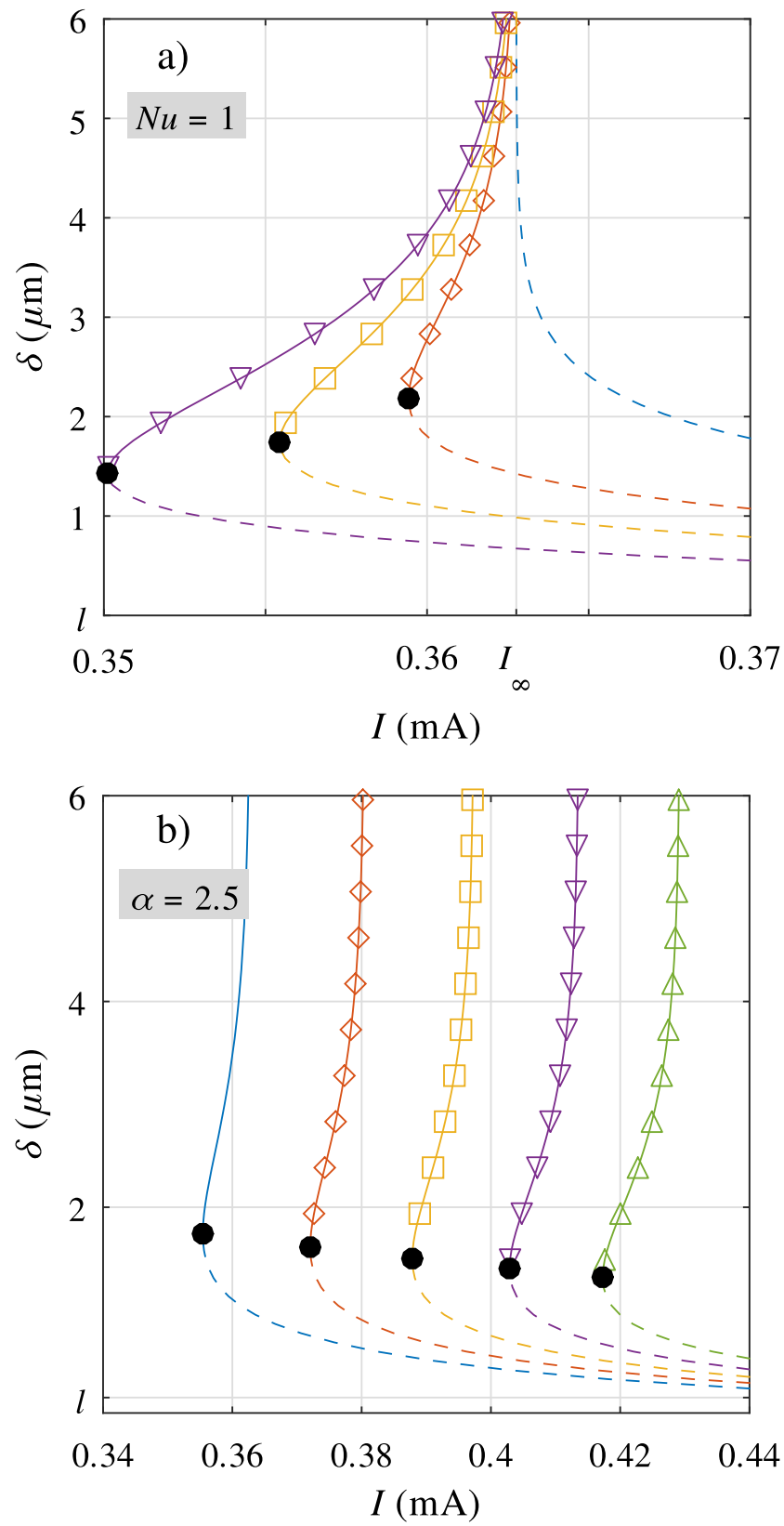

FIG. 5. (a) Half-length $\delta$ of the normal region according to Eq. (5) for $N u=1$ and various cross section ratios: $\alpha=1$ (no symbol), $\alpha=2$ (red diamond), $\alpha=2.5$ (yellow square), and $\alpha=3$ (violet triangle). (b) Half-length $\delta$ of the normal region according to Eq. (5) for $\alpha=2.5$ and various Nusselt numbers: $N u=1$ (no symbol), $N u=1.1$ (red diamond), $N u=1.2$ (yellow square), $N u=1.3$ (violet triangle), and $N u=1.4$ (green triangle). Black dots indicate the coordinates of $\left(\delta^{*}, l^{*}\right)$ for each set of $\alpha$ and Nu parameters, which are the limits between the stable (solid lines) and unstable branches (dashed lines).

in Sec. IV. The minimal stable length $\delta^{*}$ (see black dots in Fig. 5) decreases with the Nusselt number as observed experimentally, and the extent of the current range for which a stable hot spot can be observed, $I_{\infty}-I^{*}$, increases with the Nusselt number.
This model provides a robust explanation for the fact that we were not able to observe hot-spots with spatial extent as small as $l$. It shows that the best achievable resolution of this kind of sensors will depend on the Nusselt number for a given section ratio $\alpha$. This intrinsic limitation will mostly be visible for very small Nusselt numbers and shall disappear as the velocity is increased.

\section{VELOCITY FLUCTUATIONS MEASUREMENTS}

In this paragraph, after describing a specially developed electronic driver, we present some measurements in a turbulent grid flow.

\section{A. Constant voltage driver}

An electronic device was specifically designed for the purpose of driving this hot-wire. The main requirements that had to be fulfilled were the thermal stability of the hot-spot together with a fast response of the output signal.

As shown in Secs. V and IV, the wire exhibits a hysteretical behavior: if the central region happens to become superconducting during a measurement, the anemometer has to be reignited, which leads to discontinuous measurement periods. There are three common ways to drive a hot-wire: constant current, constant voltage, and constant resistance (or temperature).

Constant temperature (or resistance) anemometers are very popular due to their ability to decrease the temporal response time. This feature is mainly due to the fact that preventing temperature fluctuations is a powerful way to workaround the thermal inertia of the wire. In the case of cryogenic sensors, the specific heat of superconducting and metallic materials becomes so low that the response time is dominated by the calorific capacity of the thermal boundary layer. Thus, constant temperature operation is not as crucial at low temperature because the calibration accounts for the consequences of the wire temperature fluctuations.

Constant current anemometers are the simplest from a technological point of view. They also generally exhibit the best signal to noise ratio. However, since the sensitivity $\frac{T}{R} \frac{d R}{d T}$ of our sensor is positive, the constant current framework is possibly unstable: an increase in the velocity leads to a decrease in the mean temperature and in the overall resistance, which finally decreases the amount of dissipated power. This scenario, when combined with eventually large turbulence ratios, leads to frequent extinctions of the hot-spot.

From this point of view, constant voltage driving is selfcontrolled: an increase in the velocity leads to an increase in the dissipated power, which prevents the central region of the wire from becoming superconducting during a measurement. Another advantage of the constant voltage setup is that it tends to maintain the wire in the middle of the range of stable currents (see dashed lines in Fig. 3). Note that constant voltage (and constant temperature) anemometry can be biased by the voltage drop across the cables to the sensors. ${ }^{21}$ This effect is less pronounced for sensors with resistances of several tens of ohms, such as the present one.

We have designed a constant voltage electronic driver adapted to our wires (see Fig. 6) inspired from original works by Sarma. ${ }^{2}$

The base principle is to use a fast differential amplifier to maintain the same voltage across a reference resistor, driven with a constant current, and the hot-wire. The reference voltage across the 


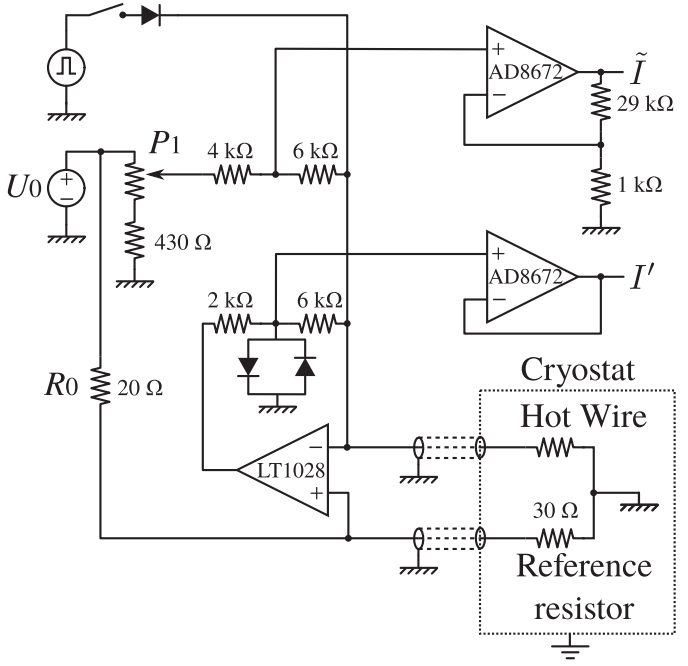

FIG. 6. Schematic of the constant voltage anemometer driver. The operational amplifiers LT 1028, which maintains the constant voltage across the sensor, is wired to produce a $6 \mathrm{k} \Omega$ transimpedance gain. For a nominal, $20 \mathrm{mV}$ voltage across the sensor, the resulting voltage gain is about $6 \mathrm{k} \Omega / R_{\text {wire }} \approx 200$. The actual circuit was etched on standard fiber-glass and copper board with a ground layer on each face and SMD resistors and diodes.

resistors can be tuned by changing the current source (a tunable reference voltage source in series with a large resistor $R_{0} \gg R_{\text {ref }}$ ) feeding the reference resistor.

The current $I$ that flows into the hot wire is composed of a stationary current $\tilde{I}$ and a fluctuating current $I^{\prime}: I=\tilde{I}+I^{\prime}$. The stationary current $\tilde{I}$ is adjusted using a potentiometer $P_{1}$ in order to minimize the time averaged fluctuating component: $\left\langle I^{\prime}\right\rangle \approx 0 \mathrm{~A}$. This way, the fluctuating component, which is generally small, can further be amplified.

The following three features were added to the original design by Sarma: ${ }^{22}$

- The reference resistor is deported as close as possible to the hot wire (inside the cryostat) so that both of them are submitted to the same environmental noise. This allows for a better noise rejection in electromagnetically noisy environments.

- A diode bridge prevents the current in the wire from reaching too large values in case the wire happens to become entirely superconducting.

- When the wire is entirely superconducting, it can be ignited using a current pulse $\delta I$. This is achieved by means of an external pulse generator connected to the current injection branch via a diode.

\section{B. Measurements in a grid flow}

A hot-wire has been tested in a high Reynolds number grid flow (TSF experiment ${ }^{23,24}$ ). This TSF experiment is a pressurized closed loop of liquid helium in which a turbulent grid flow is driven by means of a cold turbine. The temperature was maintained at $2.6 \mathrm{~K}$ with an accuracy of better than $0.1 \mathrm{~K}$. The wire was driven using the constant voltage setup described in Sec. VI. 1 min of signal was acquired directly from the driver output $\left(I^{\prime}\right)$ on a VXI-e1430 acquisition board, which features an anti-aliasing delta-sigma analog to digital converter, at a sampling frequency of $320 \mathrm{kHz}$. The voltage fluctuations span over a few volts. The Power Spectral Density (PSD) was computed numerically using Welch's algorithm with 1-s-long Hanning-filtered blocks. In Fig. 8 is shown the raw PSD of the anemometer current output $I^{\prime}$ measured using the hot wire. For comparison, the PSD of velocity obtained with a Pitot tube at the same velocity ${ }^{24}(v=1.7 \mathrm{~m} / \mathrm{s})$, and approximately the same distance from the grid ( $L \approx 480 \mathrm{~mm}$, see Fig. 7), is also shown. At this velocity, the Reynolds number based on the grid mesh size $M=3.9 \mathrm{~mm}$ and the rms velocity $v^{\prime} \approx 0.015 v$ is $R e \approx v^{\prime} M / v \approx 3700$. At such low turbulence intensity, the hot wire response to velocity fluctuations can be linearized.

At low frequency, and up to $f \approx 750 \mathrm{~Hz}$, the two spectra are comparable.

The longitudinal integral length scale of the flow is $L_{l} \approx 2.3 \mathrm{~mm}$, and the corresponding cutoff frequency is $f_{0} \approx 75 \mathrm{~Hz}$ (see Ref. 24). The hot-wire's PSD is flat for $f<f_{0}$, as expected for uncorrelated large scales of the flow. Above $f_{0}$ and up to $f \approx 3 \mathrm{kHz}$, the PSD reasonably follows a $f^{-5 / 3}$ power law and this is emphasized in the inset of Fig. 8, where we have represented the PSD compensated by $f^{5 / 3}$. The bump around $2 \mathrm{kHz}$ preceding attenuation at high frequencies corresponds to the expected bottleneck (see Refs. 25-27 and references therein).

The smallest characteristic length scale of the flow is the dissipative length scale $\eta$, and it can be estimated as $\eta=L_{l} R e^{-3 / 4}$ $\approx 5 \mu \mathrm{m}$. Such a tiny length scale is out-of-reach of commercial hot wires by typ. 2 decades, but it can be nearly resolved with the proposed hot wire, with its hot-spot length of order $2 \delta \approx 8 \mu \mathrm{m}$. As illustrated by the spectral attenuation beyond the bottleneck (see Fig. 8), this high space resolution-combined with the high time resolution imparted by the cryogenic environment-allows us to resolve nearly

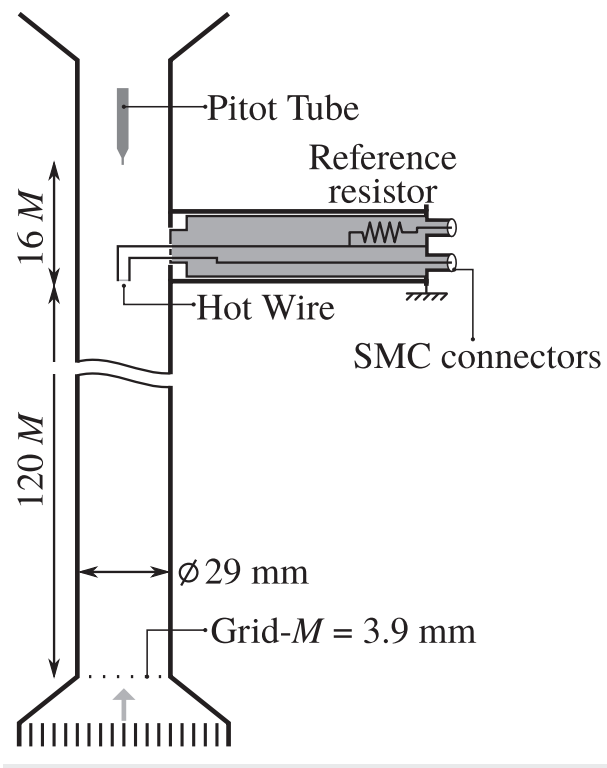

FIG. 7. Schematic of the test chamber in TSF experiment. 


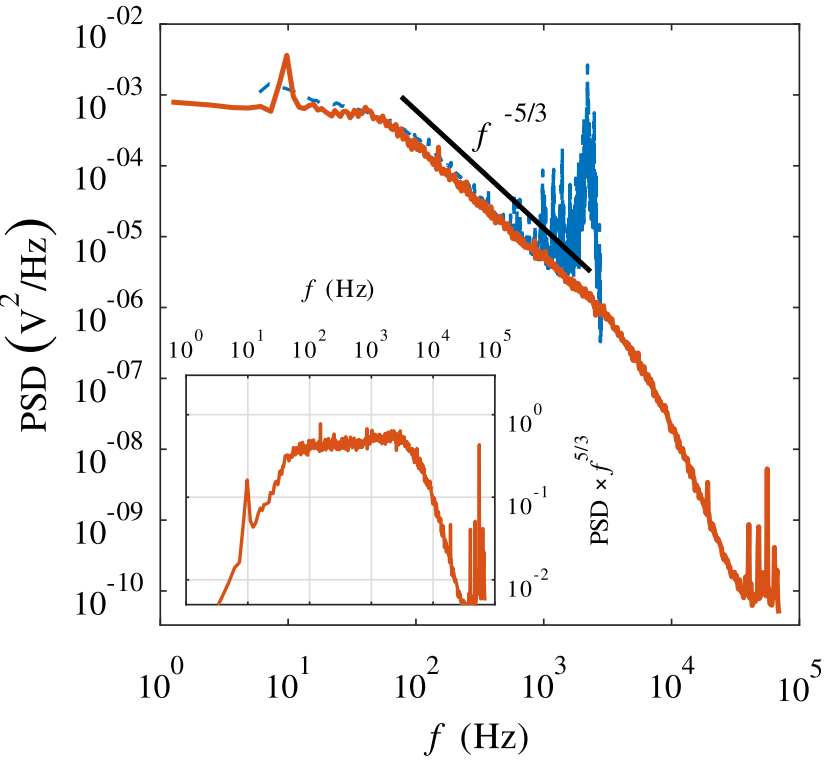

FIG. 8. Power Spectral Density (PSD) of the hot-wire driver voltage output (solid line) and the Pitot tube velocity signal in the TSF grid experiment at mean velocity $v=1.7 \mathrm{~m} / \mathrm{s}$. The Pitot PSD was shifted vertically to collapse with the hot wire PSD. Inset: PSD of the hot-wire compensated by $f^{5 / 3}$

all the dissipative scales of the turbulent flow. The electronic ground noise of the measurement system, about $V_{\text {noise }} \approx 10 \mu \mathrm{V} / \mathrm{Hz}^{1 / 2}$, is reached at frequency $f_{\text {noise }} \approx 40 \mathrm{kHz}$, after more than one frequency decade of dissipative range. The corresponding signal to noise ratio can be estimated as the standard deviation of the signal divided by $V_{\text {noise }} \times \sqrt{f_{\text {noise }}}$ leading to a value of 140 .

\section{CONCLUSION AND DISCUSSION}

The design and first implementation of a new type of monolithic superconducting hot-wire anemometer has been presented. The main advantage - as compared to previous designs-is a smaller number of fabrication steps. Even though the diameter of those sensors can be ten times smaller than previous models, their resolution has been shown to be of order $5 \mu \mathrm{m}$, that is, comparable to the most-resolved hot-wires reported in the literature.

It was shown experimentally that the minimal length of the sensitive hot-spot is generally much larger than the length where the wire has a reduced section area, preventing the measurements of eddies of submicrons sizes. We derived a simple model that shows this limitation cannot be overcome easily since at low velocity, very short hot-spots are intrinsically unstable. The only control parameter in the fabrication of those wires is the section reduction ratio, and the model shows that the larger the section reduction, the smaller the achievable hot-spot. Obviously, however, the section cannot be reduced much further, at least without introducing in the fabrication process some strengthening steps to preserve mechanical resistance of the wire.

Using a lab-made constant voltage controller driving the sensor, we have obtained some first turbulent measurements in stringent flow conditions: a helium grid flow with an integral length scale of only few millimeter and a Kolmogorov length scales below $10 \mu \mathrm{m}$. These first results demonstrate that a satisfactory signal-tonoise ratio can be obtained, even in a flow where the turbulence intensity is below $2 \%$, and thus even when velocity fluctuations very small.

\section{ACKNOWLEDGMENTS}

This work was supported by the French National Research Agency, Grant No. ANR-05-BLAN-0316. We would like to thank J. Salort and collaborators for providing the Pitot tube dataset, A. Sulpice for providing us with various superconducting cables samples, J.-L. Bret and C. Guttin for helping with the design of the constant voltage anemometer, and all members of the TSF collaboration for their support. Special thanks go to B. Castaing and B. Rousset for fruitful discussions.

\section{REFERENCES}

${ }^{1}$ S. Pope, Turbulent Flows (Cambridge University Press, 2000).

${ }^{2}$ S. Van Sciver, Helium Cryogenics, International Cryogenics Monograph Series (Springer, 2012).

${ }^{3}$ B. Rousset, P. Bonnay, P. Diribarne, A. Girard, J. Poncet, E. Herbert, J. Salort, C. Baudet, B. Castaing, L. Chevillard, F. Daviaud, B. Dubrulle, Y. Gagne, M. Gibert, B. Hébral, T. Lehner, P.-E. Roche, B. Saint-Michel, and M. Bon Mardion, "Superfluid high Reynolds von Kármán experiment,” Rev. Sci. Instrum. 85, 103908 (2014).

${ }^{4}$ E. Rusaouen, B. Chabaud, J. Salort, and P.-E. Roche, "Intermittency of quantum turbulence with superfluid fractions from 0\% to 96\%," Phys. Fluids 29, 105108 (2017).

${ }^{5}$ J. Maurer, P. Tabeling, and G. Zocchi, "Statistics of turbulence between two counterrotating disks in low-temperature helium gas," Europhys. Lett. 26, 31-36 (1994).

${ }^{6}$ O. Chanal, B. Chabaud, B. Castaing, and B. Hébral, "Intermittency in a turbulent low temperature gaseous helium jet," Eur. Phys. J. B 17, 309-317 (2000).

${ }^{7}$ S. Pietropinto, C. Poulain, C. Baudet, B. Castaing, B. Chabaud, Y. Gagne, B. Hébral, Y. Ladam, P. Lebrun, O. Pirotte, and P.-E. Roche, "Superconducting instrumentation for high Reynolds turbulence experiments with low temperature gaseous helium," Physica C 386, 512 (2003).

${ }^{8}$ S. C. Bailey, G. J. Kunkel, M. Hultmark, M. Vallikivi, J. P. Hill, K. A. Meyer, C. Tsay, C. B. Arnold, and A. J. Smits, "Turbulence measurements using a nanoscale thermal anemometry probe," J. Fluid Mech. 663, 160-179 (2010).

${ }^{9}$ E. Bodenschatz, G. P. Bewley, H. Nobach, M. Sinhuber, and H. Xu, "Variable density turbulence tunnel facility," Rev. Sci. Instrum. 85, 093908 (2014).

${ }^{10}$ B. Castaing, B. Chabaud, and B. Hebral, "Hot wire anemometer operating at cryogenic temperatures,” Rev. Sci. Instrum. 63, 4167-4173 (1992).

${ }^{11}$ O. Chanal, B. Baguenard, O. Béthoux, and B. Chabaud, "Micronic-size cryogenic thermometer for turbulence measurements," Rev. Sci. Instrum. 68, 2442-2446 (1997).

${ }^{12}$ Y. Fan, G. Arwatz, T. W. Van Buren, D. E. Hoffman, and M. Hultmark, "Nanoscale sensing devices for turbulence measurements," Exp. Fluids 56, 138 (2015).

${ }^{13}$ M. K. Fu, Y. Fan, and M. Hultmark, "Design and validation of a nanoscale crosswire probe (x-nstap)," Exp. Fluids 60, 99 (2019).

${ }^{14}$ Y. Borisenkov, G. Gulitski, M. Kholmyansky, S. Krylov, A. Liberzon, and A. Tsinober, "Micro-machined super-miniature hot-film multi-array probe for field experiments with sub-Kolmogorov resolution," J. Turbul. 16, 525-539 (2015).

${ }^{15}$ J. Valentin, E. Rusaouën, B. Chabaud, O. Pirotte, and P.-E. Roche, "Array of ultra-miniaturized hot-films for high Reynolds number flows," in 16th European Turbulence Conference, 21-24 August 2017, Stockholm, 2017. 
${ }^{16} \mathrm{~L}$. Dresner, in symposium on Superconductor Stability ["Superconductor stability '90: A review," Cryogenics 31, 489-498 (1991)].

${ }^{17}$ B. Castaing, B. Chabaud, F. Chilla, B. Hebral, A. Naert, and J. Peinke, "Anemometry in gaseous he-4 around 4-k," J. Phys. III 4, 671-674 (1994).

${ }^{18}$ D. Durì, C. Baudet, J.-P. Moro, P.-E. Roche, and P. Diribarne, "Hot-wire anemometry for superfluid turbulent coflows," Rev. Sci. Instrum. 86, 025007 (2015).

${ }^{19} \rho_{\mathrm{NbTi}}=0.610^{-6} \Omega \mathrm{m}, k_{\mathrm{NbTi}}=0.6 \mathrm{~W} \mathrm{~m}^{-1} \mathrm{~K}^{-1}, T_{c}=9 \mathrm{~K}, k_{\mathrm{He}}=0.02 \mathrm{~W} \mathrm{~m}^{-1} \mathrm{~K}^{-1}$.

${ }^{20}$ Y. Bychkov, R. Herzog, and I. Khukhareva, "Thermal conductivity and electrical resistivity of NbTi alloys at low temperatures," Cryogenics 21, 741-745 (1981).

${ }^{21}$ P. Ligeza, "A four-point constant-current/temperature controlled circuit for anemometric applications," Rev. Sci. Instrum. 71, 109-112 (2000).

${ }^{22}$ G. R. Sarma, "Transfer function analysis of the constant voltage anemometer," Rev. Sci. Instrum. 69, 2385-2391 (1998).
${ }^{23}$ P. Diribarne, J. Salort, C. Baudet, B. Belier, B. Castaing, L. Chevillard, F. Daviaud, S. David, B. Dubrulle, and Y. Gagne, and others, "TSF experiment for comparision of high Reynolds number turbulence in He I and He II: First results," in Advances in Turbulence XII (Springer, 2009), pp. 701-704.

${ }^{24}$ J. Salort, C. Baudet, B. Castaing, B. Chabaud, F. Daviaud, T. Didelot, P. Diribarne, B. Dubrulle, Y. Gagne, F. Gauthier et al., "Turbulent velocity spectra in superfluid flows," Phys. Fluids 22, 125102 (2010).

${ }^{25}$ D. A. Donzis and K. R. Sreenivasan, "The bottleneck effect and the Kolmogorov constant in isotropic turbulence," J. Fluid Mech. 657, 171-188 (2010).

${ }^{26}$ T. Ishihara, K. Morishita, M. Yokokawa, A. Uno, and Y. Kaneda, "Energy spectrum in high-resolution direct numerical simulations of turbulence," Phys. Rev Fluids 1, 082403 (2016)

${ }^{27}$ C. Küchler, G. Bewley, and E. Bodenschatz, "Experimental study of the bottleneck in fully developed turbulence,” J. Stat. Phys. 175, 617 (2019). 